\title{
Resin adhesion strengths to zirconia ceramics after primer treatment with silane coupling monomer or oligomer
}

\author{
Masahiro OKADA ${ }^{1}$, Kazusa INOUE${ }^{1}$, Masao IRIE ${ }^{1}$, Hiroaki TAKETA², Yasuhiro TORII² and Takuya MATSUMOTO ${ }^{1}$ \\ ${ }^{1}$ Department of Biomaterials, Graduate School of Medicine, Dentistry and Pharmaceutical Sciences, Okayama University, 2-5-1 Shikata-cho, Kita-ku, \\ Okayama 700-8558, Japan \\ ${ }^{2}$ Department of Comprehensive Dentistry, Okayama University Hospital, 2-5-1 Shikata-cho, Kita-ku, Okayama 700-8558, Japan \\ Corresponding author, Takuya MATSUMOTO; E-mail: tmatsu@md.okayama-u.ac.jp
}

\begin{abstract}
Resin bonding to zirconia ceramics is difficult to achieve using the standard methods for conventional silica-based dental ceramics, which employ silane coupling monomers as primers. The hypothesis in this study was that a silane coupling oligomer -a condensed product of silane coupling monomers - would be a more suitable primer for zirconia. To prove this hypothesis, the shear bond strengths between a composite resin and zirconia were compared after applying either a silane coupling monomer or oligomer. The shear bond strength increased after applying a non-activated ethanol solution of the silane coupling oligomer compared with that achieved when applying the monomer. Thermal treatment of the zirconia at $110^{\circ} \mathrm{C}$ after application of the silane coupling agents was essential to improve the shear bond strength between the composite resin cement and zirconia.
\end{abstract}

Keywords: Silane coupling, Oligomer, Zirconia, Bonding strength, Thermal treatment

\section{INTRODUCTION}

Zirconia-based ceramics have attracted great interest in prosthetic and implant dentistry because of their superior mechanical performance (such as strength, toughness, and fatigue resistance) ${ }^{1-3)}$. The major clinical problem with the use of zirconia-based ceramics is the difficulty in achieving suitable adhesion with intended synthetic substrates or natural tissues ${ }^{4,5}$.

Adhesion between organic and inorganic materials is often achieved using silane coupling agents, which are organofunctional alkoxysilane esters typically with three hydrolyzable alkoxy groups on the silicon atom. The application of silane coupling agents is widely accepted for promoting adhesion with silica-based ceramics, and they are used in clinical work in prosthetic dentistry6). However, traditional silane coupling agents such as $\gamma$-methacryloxypropyl-trimethoxysilane $(\gamma \text {-MPS })^{7}$ are not truly effective with zirconia-based ceramics, as zirconia possesses a relatively non-polar surface and is more chemically stable than silica-based ceramics ${ }^{4}$.

Several uncommon silane coupling agents have been screened for zirconia-based ceramics: 3-acryloxypropyltrimethoxysilane ${ }^{8)}$ 3-isocyanatopropyltriethoxysilane ${ }^{8)}$, 3-glycidoxypropyltrimethoxysilane ${ }^{9)}$, styrylethyltrimethoxysilane ${ }^{9)}$, and 3-mercaptopropyltrimethoxysilane ${ }^{10)}$. These silane coupling agents have one organofunctional group in their molecular structures, and there have been no reports on the effect of the number of organofunctional groups of the silane coupling agent on adhesion. One of the ways to increase the number of organofunctional groups is the use of silane coupling oligomer. Silane coupling oligomers are condensed products of silane coupling monomers and generally consist of dimers and trimers (with molar mass up to approximately $1,000 \mathrm{~g} / \mathrm{mol})^{11}$. They contain almost no silanols, which gives them a long shelf life, and have lower volatility because of their higher molecular weight compared with silane coupling monomers. In addition, silane coupling oligomers release less alcohol when curing than monomers.

The objective of the present study was to evaluate the bond strengths between resin cements and zirconia ceramics after primer treatments with a silane coupling oligomer (Fig. 1). The hypothesis in this study was that the bond strength to zirconia ceramics after applying the silane coupling oligomer would be higher than that when applying the monomer. This hypothesis was arrived at because the oligomer has a larger number of polymerizable methacryloyloxy groups per molecule, making it effective for use with the zirconia surface, which consists of a low density of hydroxyl groups ${ }^{12,13)}$. Furthermore, the effects of acid activation before the application of silane coupling agents and heat treatment after the application were also investigated, because these factors are reported to be effective for the formation $\mathrm{Si}-\mathrm{O}$-substrate linkage in the case of silane coupling monomers for $\mathrm{SiO}_{2}$ substrates ${ }^{7}$. The results showed that the application of non-activated silane coupling oligomer solution showed improved bond strength between resin cements and zirconia due to the formation of thicker silane coupling layer as compared with silane coupling monomer solution. The thickness of silane coupling layers was estimated from attenuated total reflection Fourier-transform infrared (ATR FT-IR) spectroscopy measurements. Finally, in order to improve the bond strength by forming thicker silane coupling layer, the application of pristine silane coupling agent (without dilution) was examined. 


\section{MATERIALS AND METHODS}

\section{Materials}

The materials used in this study are listed in Table 1. A 95.0 vol\% ethanol solution with purified water with a specific resistance of $18.2 \mathrm{M} \Omega \cdot \mathrm{cm}$ (Milli-Q water, Millipore, Bedford, MA, USA) was prepared and allowed to stabilize for $24 \mathrm{~h}$ at room temperature. In the first experiment using silane solutions, 1.0 vol\% solutions of the silane coupling agents were prepared with pure ethanol or a $\mathrm{pH}$-adjusted ethanol/water solution (the $\mathrm{pH}$ was adjusted to 4.5 using $1 \mathrm{M}$ acetic acid) in 50-mL polyethylene bottles. For the $\mathrm{pH}$-adjusted ethanol/water solution (i.e., the activated solution), the silane solutions were used immediately after being allowed to hydrolyze for $1 \mathrm{~h}$ at room temperature. In the second experiment (a) Monomer

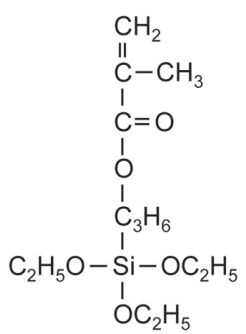

(b) Oligomer

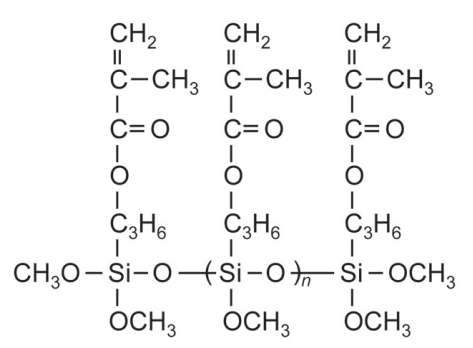

Fig. 1 Chemical structures of silane coupling monomer (a) and oligomer (b) used in this study.

The degree of condensation, $n$, in (b) was calculated to be 1.86 from the alkoxy group content of the product (22 wt\%). using pristine silane coupling agents, the silane coupling monomer and oligomer were used as received without dilution with solvents.

\section{Specimen preparation}

A low-speed cutting machine was used to cut the 3 mol\% yttria-stabilized tetragonal zirconia into samples with dimensions of approximately $6 \times 6 \times 2 \mathrm{~mm}$, which were then embedded in an epoxy resin (SpeciFix 20 Kit, Struers, Rodovre, Denmark). The surfaces of the zirconia were ground with \#600 silicon carbide abrasive paper (Struers) under water irrigation to remove pollutants and to obtain a flat uniform surface in the same manner as described previously ${ }^{14,15)}$.

In the first experiment using the silane solutions, the specimens were divided randomly into eight groups (Groups I-IV with monomer or oligomer; each $n=5$ ) based on the treatment procedure used for the zirconia surface: application of silane solution without acetic acid and no thermal treatment (Group I); application of silane solution without acetic acid followed by thermal treatment (Group II); application of silane solution with acetic acid and no thermal treatment (Group III); or application of silane solution with acetic acid followed by thermal treatment (Group IV). In the above procedure, $20 \mu \mathrm{L}$ of the silane solution (1.0 vol\%) of monomer or oligomer was applied on each zirconia surface, and then, the solution was blown with air $(0.5 \mathrm{MPa} ; 10 \mathrm{~s})$. For the thermal treatment, after applying the solution and blowing the sample with air, it was placed in an electric furnace set to $110^{\circ} \mathrm{C}$ for $10 \mathrm{~min}$, followed by cooling at room temperature for $30 \mathrm{~min}$. Resin luting material (Panavia V5) was then filled in a Teflon mold having a cylindrical hollow (3.6-mm diameter and 2.0-mm depth) on the zirconia surface using a syringe tip before being

Table 1 Materials used in this study

\begin{tabular}{|c|c|c|}
\hline Product name & Composition & Manufacturer \\
\hline Zirconia specimen: Lava & $3 \mathrm{~mol} \%$ yttria-stabilized tetragonal zirconia & $\begin{array}{l}\text { 3M ESPE Dental Products, } \\
\text { Seefeld, Germany }\end{array}$ \\
\hline Resin cement: Panavia V5 & $\begin{array}{l}\text { Base resin: bisphenol-A-glycidyl methacrylate, } \\
\text { triethyleneglycol dimethacrylate, and other } \\
\text { methacrylate monomers } \\
\text { Filler: surface-modified barium glass, } \\
\text { surface-modified aluminum fluoride silicate glass, } \\
\text { and silica microfiller }\end{array}$ & $\begin{array}{l}\text { Kuraray Noritake Dental, } \\
\text { Tokyo, Japan }\end{array}$ \\
\hline $\begin{array}{l}\text { Silane coupling monomer: } \\
\text { KBE-503 }\end{array}$ & $\gamma$-Methacryloxypropyl triethoxysilane & $\begin{array}{l}\text { Shin-Etsu Chemical, } \\
\text { Tokyo, Japan }\end{array}$ \\
\hline $\begin{array}{l}\text { Silane coupling oligomer: } \\
\text { X-40-9296 }\end{array}$ & $\begin{array}{l}\text { Condensed product of } \gamma \text {-methacryloxypropyl } \\
\text { trimethoxysilane } \\
\text { Alkoxy group content: } 22 \mathrm{wt} \% \\
\text { Metacryl equivalent: } 230 \mathrm{~g} / \mathrm{mol}\end{array}$ & Shin-Etsu Chemical \\
\hline Solvent: Ethanol & Ethanol (99.5 vol\%) & $\begin{array}{l}\text { Wako Pure Chemical Industries, } \\
\text { Osaka, Japan }\end{array}$ \\
\hline Acid: Acetic acid & Acetic acid $(99.7 \%)$ & Wako Pure Chemical Industries \\
\hline
\end{tabular}


polymerized using a light curing unit $\left(2,000 \mathrm{~mW} / \mathrm{cm}^{2}\right.$; Bluephase 20i; Ivoclar Vivadent, Schaan, Liechtenstein) for $20 \mathrm{~s}$. After polymerization, the specimens were stored in distilled water at $37^{\circ} \mathrm{C}$ for 7 days.

In the second experiment (Group V with monomer or oligomer; each $n=5$ ) using pristine silane coupling agents, $10 \mu \mathrm{L}$ of the silane coupling agent (without dilution and without acetic acid) was directly applied to each zirconia surface and then blown with air (0.5 MPa; $10 \mathrm{~s})$, and then, $200 \mu \mathrm{L}$ of pure ethanol was applied and blown with air $(0.5 \mathrm{MPa} ; 10 \mathrm{~s})$ to remove the excess silane coupling agent. The removal procedure was repeated five times. The thermal treatment, resin luting, and storage were conducted in the same manner as that described for the first experiment.

\section{Shear bond strength measurements}

The shear bond strengths were determined after 7-day storage in distilled water at $37^{\circ} \mathrm{C}$. The specimens were mounted on a testing machine (Autograph AG-X, Shimadzu, Kyoto, Japan), and shear stress was applied onto one side of the cylindrical resin cement on each zirconia surface at a constant speed of $0.5 \mathrm{~mm} / \mathrm{min}$, in the same manner as described previously ${ }^{14,15)}$.

After the normality and the homogeneity of variance were tested using Shapiro-Wilk and Levene's tests, respectively, a three-way analysis of variance (ANOVA) was performed for first experimental Groups I-IV (factors: acid activation, heat treatment and type of silane coupling agent). The Tukey-Kramer test was used to detect multiple comparisons among the above experimental groups. For the additionally conducted experimental Group V (with monomer and oligomer, respectively), Student's $t$ test was performed with the Group II. All statistical tests were performed using R ver 3.3.2 ${ }^{16)}$ at preset alpha levels of 0.05 .

\section{Surface compositions}

ATR FT-IR spectra of the intact silane coupling monomer, oligomer, and solvent (ethanol) were recorded after applying the liquids to a ZnSe prism. An IRAffinity1S (Shimadzu) was used at $4 \mathrm{~cm}^{-1}$ resolution with 64 scans at room temperature (approximately $25^{\circ} \mathrm{C}$ ). ATR FT-IR spectroscopy spectra of the zirconia after the primer treatments were also obtained after pressing the substrate on the $\mathrm{ZnSe}$ prism.

\section{RESULTS}

\section{Application of silane solution}

First, a 1 vol\% solution of the silane coupling monomer or oligomer was used as a primer for zirconia, and the effects of the type of silane coupling agent (i.e., comparison of the monomer and oligomer), acetic acid dissolved in the ethanol solution (i.e., comparison of acid activation), and heat treatment after primer application were investigated. Table 2 summarized the shear bond strengths between the zirconia and resin after 7-day water storage at $37^{\circ} \mathrm{C}$. Three-way ANOVA revealed statistically significant differences for "the type of silane coupling agent ( $p=0.0332)$ " and "heat treatment $\left(p=4.76 \times 10^{-6}\right)$ " factors. Conversely, the statistical analysis revealed no significant difference for the "acid activation ( $p=0.608)$ " factor. Only the double interaction between "the type of silane coupling agent and heat treatment" factors was significant $(p=0.00465)$. The triple interaction was not statistically significant $(p=0.191)$. Note that the Tukey-Kramer test revealed significant differences for the Group II treatment (without acid activation but with thermal treatment) with oligomer.

\section{ATR FT-IR spectroscopy measurements}

In order to understand the reason why the oligomer solution showed improved bond strength, the thickness of silane coupling layers on the zirconia substrate were estimated from ATR FT-IR spectroscopy. Figure 2 presents the ATR FT-IR spectroscopy spectra of the intact liquids (the ethanol used as a solvent and silane coupling monomer and oligomer) and zirconia surface directly after grinding and washing. In the spectra of the intact silane coupling agents in Fig. 2(b) and (c), characteristic peaks originating from ester groups are observed at 1,720,1,320,1,297, 1,192, and 1,167 $\mathrm{cm}^{-1}$, and several $\mathrm{C}-\mathrm{H}$ stretching vibration modes are observed at 2,840-3,000 $\mathrm{cm}^{-1}{ }^{17,18)}$. Vinyl $\mathrm{C}=\mathrm{C}$ stretching vibrations are observed at $1,638 \mathrm{~cm}^{-1}$ for both types of silane coupling agents; the broadened bands at approximately $1,087 \mathrm{~cm}^{-1}$ for the silane coupling oligomer are assigned to $\mathrm{Si}-\mathrm{O}-\mathrm{Si}$ anti-symmetric stretching vibration, which

Table 2 Shear bond strengths between composite resin cements and zirconia treated with 1 wt\% ethanol solution of silane coupling agents.

\begin{tabular}{lccccc}
\hline & Group I & Group II & Group III & Group IV \\
\hline Acid activation & - & - & + & + \\
Heat treatment & Monomer & $1.05 \pm 0.67^{\mathrm{a}}$ & $1.75 \pm 0.61^{\mathrm{a}, \mathrm{b}}$ & $1.77 \pm 0.96^{\mathrm{a}, \mathrm{b}}$ & $4.05 \pm 1.80^{\mathrm{a}, \mathrm{c}}$ \\
$\begin{array}{l}\text { Silane coupling agent } \\
\text { (solution) }\end{array}$ & Oligomer & $0.99 \pm 1.04^{\mathrm{a}}$ & $6.97 \pm 3.23^{\mathrm{c}}$ & $0.89 \pm 0.37^{\mathrm{a}}$ & $5.25 \pm 3.16^{\mathrm{b}, \mathrm{c}}$ \\
\hline
\end{tabular}

Mean $(\mathrm{MPa}) \pm \mathrm{SD}$

Different subscript letters indicate statistically significant difference $(p<0.05)$. 

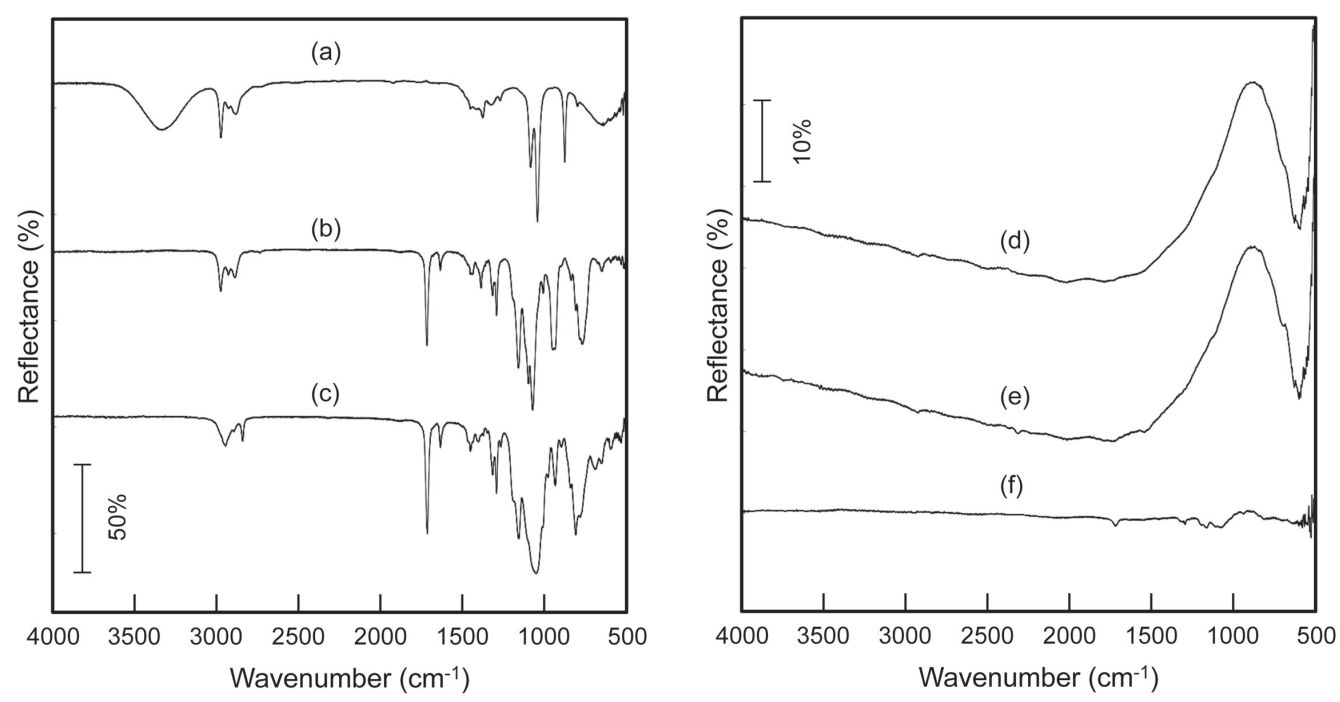

Fig. 2 ATR FT-IR spectroscopy spectra of (a) ethanol used as a solvent, (b) pristine silane coupling monomer, (c) pristine silane coupling oligomer, and zirconia surface (d) after grinding (before application of silane solutions) and after application of silane solutions (1 vol\%) of (e) monomer and (f) oligomer followed by blowing with air.

confirms the formation of siloxane oligomers ${ }^{17}$.

For the intact zirconia before application of the silane solutions, the absorption bands at approximately $520 \mathrm{~cm}^{-1}$ in Fig. 2(d) correspond to $\mathrm{Zr}-\mathrm{O}$ vibrations ${ }^{19}$, and obvious changes were not observed for the zirconia substrate after application of the $1.0 \mathrm{vol} \%$ solution of the silane coupling monomer (after air blowing and without thermal treatment), as shown in Fig. 2(e). In contrast, when applying the silane coupling oligomer under the same conditions, as observed in Fig. 2(f), the absorption bands at approximately $520 \mathrm{~cm}^{-1}$ decreased, and several peaks associated with silane coupling oligomer are observed at $1,720 \mathrm{~cm}^{-1}(\mathrm{C}=\mathrm{O}$ groups $)$ and at approximately $1,087 \mathrm{~cm}^{-1}$ (Si-O-Si group).

\section{Direct application of silane coupling agent}

To increase the amount of silane coupling agents on the zirconia substrate and to evaluate the interaction between non-activated silane coupling agents and zirconia substrates, the pristine silane coupling agents were applied directly (without diluting the solvent), and the excess amount of silane coupling agents was removed by washing with ethanol. In the ATR FT-IR spectra of the zirconia surfaces with the silane coupling monomer or oligomer applied, the peaks due to the silane coupling agents decreased after washing. The degree of remaining silane coupling agent on the zirconia surface was estimated from the ATR FT-IR spectroscopy peak intensity ratios calculated from the intensity of the peak at $1,720 \mathrm{~cm}^{-1}(\mathrm{C}=\mathrm{O}$ groups $)$ of the silane coupling agents on the zirconia surface and that of the pristine silane coupling agents measured directly on the ATR crystal. Before washing, the peak intensity ratio for the oligomer was higher than that of the monomer (Fig. 3). The peak intensity ratio decreased significantly after one-time

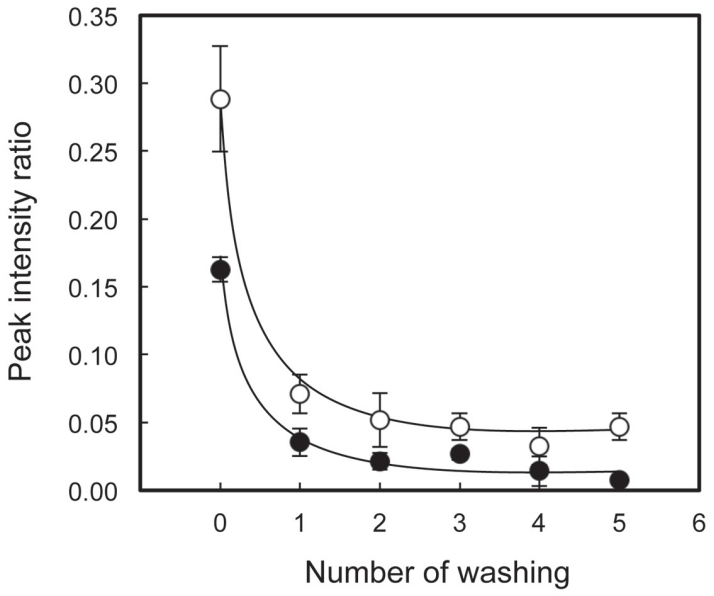

Fig. 3 Variations of ATR FT-IR spectroscopy peak intensity ratios after washing with ethanol $(n=3)$. Closed circles indicate the silane coupling monomer, and open circles indicate the oligomer. Each peak intensity ratio was calculated from the intensity of the peak at $1,720 \mathrm{~cm}^{-1}(\mathrm{C}=\mathrm{O}$ groups $)$ of the silane-applied zirconia surface and the pristine silane coupling agents measured directly on the ATR crystal.

washing. After two-time washing, the peak intensity ratio reached a plateau value and showed almost constant values until five-time washing. The plateau value for the oligomer was lager than that of monomer.

Table 3 shows the shear bond strengths between the resin and zirconia on which the pristine silane coupling agents were directly applied and then washed with ethanol five times followed by the heat treatment 
Table 3 Shear bond strengths between composite resin cements and zirconia treated with non-activated pristine silane coupling agents without diluting, followed by washing with ethanol five times.

\begin{tabular}{clc}
\hline & & Group V \\
\hline \multirow{2}{*}{ Silane coupling agent } & Monomer & $8.06 \pm 0.71^{*}$ \\
& Oligomer & $8.07 \pm 1.32^{\text {N.S. }}$ \\
\hline
\end{tabular}

Mean $(\mathrm{MPa}) \pm \mathrm{SD}$

${ }^{*} p<0.05$ vs. Group II with monomer solution.

N.S. $p>0.05$ vs. Group II with oligomer solution.

at $110^{\circ} \mathrm{C}$. The shear bond strength in the case of the pristine monomer significantly increased compared with that of the monomer solution $(p<0.05)$. In the case of oligomers, significant difference was not observed between pristine and solution states $(p>0.05)$.

\section{DISCUSSION}

The general formula of a silane coupling agent is $\mathrm{X}-\left(\mathrm{CH}_{2}\right)_{\mathrm{m}}-\mathrm{Si}(\mathrm{OR})_{3}$, where $\mathrm{X}$ is an organofunctional group that reacts with the organic matrix, $\left(\mathrm{CH}_{2}\right)_{\mathrm{m}}$ is a linker group, and OR is an alkoxy group ${ }^{7}$. The silane coupling agents must be activated by hydrolysis $(\mathrm{SiOR} \rightarrow \mathrm{SiOH})$ to form (1) hydrogen bonding to the inorganic substrate surface at ambient temperature and (2) Si-O-substrate linkage via the condensation reaction between silanol $(-\mathrm{SiOH})$ groups of silane coupling agent and hydroxyl $(-\mathrm{OH})$ groups on the substrate, which is accelerated by thermal treatment at $110-150^{\circ} \mathrm{C}^{20)}$. Therefore, twocomponent silane solutions or pre-activated silane solutions (i.e., mixtures of the silane coupling monomer, acid, water and various polar and non-polar solvents) are used in dentistry ${ }^{17)}$. Although the function of the acid is to activate (hydrolyze) alkoxysilyl groups, silanols can begin to condense with each other, forming higher-molecular-weight oligomers/polymers over time in the presence of acid in the medium. Anagnostopoulos et al. demonstrated that pre-activated silane solution exhibited a higher rate of hydrolysis compared with a two-component one; the down side is that the formation of higher-molecular-weight oligomers/polymers could reduce the effectiveness of the solution in the longer term ${ }^{21)}$. Other studies have reported enhanced bond strengths of resin to porcelain ceramics using freshly prepared two-component silane solutions ${ }^{22,23)}$.

In this study, the freshly prepared and acidactivated $1 \mathrm{vol} \%$ ethanol solution of the silane coupling monomer was inefficient as a primer for zirconia, which is consistent with previous works ${ }^{4)}$. The ATR FT-IR measurements indicate that one of the reasons for the inefficient bonding strength is the insufficient formation of the silane coupling layer on the zirconia surface (Fig. $2(\mathrm{e})$ ). This result appears to be due to less formation of hydrogen bonding between the silanol groups of the silane coupling monomer and the zirconia surface at ambient temperature; hence, most of the silane coupling monomers, which did not adsorb on the zirconia surface, were removed upon air blowing. The direct application of the silane coupling monomer without diluting with solvent followed by washing and heat treatment was effective, suggesting that the formation of a relatively thick silane coating layer is important to improve the bonding strength between the resin and zirconia. Note that the penetration depth in ATR FT-IR spectroscopy measurements, which depends on the refractive indices of the ATR crystal and sample and the infrared wavelength, ranges from several hundred nanometers to several microns for organic materials ${ }^{24}$. In contrast, the thickness of the silane coupling layer after direct application followed by washing five times is estimated to only be approximately several tens of nanometers based on the peak intensity ratio observed in Fig. 3.

For the silane coupling oligomer, the non-activated solution (without acid) significantly improved the bond strength after heat treatment at $110^{\circ} \mathrm{C}$ (Group II). The ATR FT-IR spectroscopy measurements indicated that the formation of a relatively thick silane coating layer is important to improve the bonding strength between the resin and zirconia (the thickness is estimated to range from several tens to several hundreds of nanometers). The formation of the relatively thick silane coating for the oligomer solution may stem from its larger molecular weight (i.e., low diffusion constant and larger adsorption energy) compared with that of the monomer. It is also speculated that the non-activated oligomers adsorbed on the relatively hydrophobic zirconia surface (with a water contact angle of $65^{\circ 25)}$ ) through hydrophobic interaction. Note that acid activation for the oligomer solution did not affect the bond strength, suggesting that the oligomer solution could be used as a single solution with long-term storage stability.

A phosphate-containing monomer, 10-methacryloyloxydecyldihydrogen-phosphate (MDP), is reported to form a good bond with zirconia-based ceramics $^{26)}$. The combination of MDP and a silane coupling agent to enhance the bonding of MDP resin cements has produced positive results compared with MDP-containing cements alone ${ }^{27-29)}$. Therefore, the results obtained in this study will also be important to improving MDP-containing bonding/silane primer systems. Note that heat treatment after application of the silane coupling agent is a key factor in improving the bond strength to the resin; therefore, it is necessary to develop a new device for feasible heat treatment in chair-side procedures. The findings in this study will also be applicable for primers for zirconia-based hybrid 
ceramics such as zirconia/apatite ${ }^{30,31)}$ and surface modification of zirconia fillers for resin composites ${ }^{32-34)}$.

\section{CONCLUSIONS}

The shear bond strength after applying a non-activated ethanol solution of silane coupling oligomers increased compared with that obtained after applying the silane coupling monomer. Thermal treatment at $110^{\circ} \mathrm{C}$ of zirconia after applying silane coupling agents was essential to improve the shear bond strength of the composite resin cement to the zirconia.

\section{ACKNOWLEDGMENTS}

This work was supported in part by the Japan Society for the Promotion of Science KAKENHI Grant Numbers JP25293402, JP15K13303, and JP15K15723.

\section{REFERENCES}

1) Apratim A, Eachempati P, Krishnappa Salian KK, Singh V, Chhabra S, Shah S. Zirconia in dental implantology: A review. J Int Soc Prev Community Dent 2015; 5: 147-156.

2) Pjetursson BE, Sailer I, Makarov NA, Zwahlen M, Thoma DS. All-ceramic or metal-ceramic tooth-supported fixed dental prostheses (FDPs)? A systematic review of the survival and complication rates. Part II: Multiple-unit FDPs. Dent Mater 2015; 31: 624-639.

3) Sailer I, Makarov NA, Thoma DS, Zwahlen M, Pjetursson BE. All-ceramic or metal-ceramic tooth-supported fixed dental prostheses (FDPs)? A systematic review of the survival and complication rates. Part I: Single crowns (SCs). Dent Mater 2015; 31: 603-623.

4) Thompson JY, Stoner BR, Piascik JR, Smith R. Adhesion/ cementation to zirconia and other non-silicate ceramics: where are we now? Dent Mater 2011; 27: 71-82.

5) Tzanakakis E-GC, Tzoutzas IG, Koidis PT. Is there a potential for durable adhesion to zirconia restorations? A systematic review. J Prosthet Dent 2016; 115: 9-19.

6) Blatz MB, Sadan A, Kern M. Resin-ceramic bonding: A review of the literature. J Prosthet Dent 2003; 89: 268-274.

7) Lung CYK, Matinlinna JP. Aspects of silane coupling agents and surface conditioning in dentistry: An overview. Dent Mater 2012; 28: 467-477.

8) Matinlinna JP, Heikkinen T, Ozcan M, Lassila LVJ, Vallittu PK. Evaluation of resin adhesion to zirconia ceramic using some organosilanes. Dent Mater 2006; 22: 824-831.

9) Matinlinna JP, Lassila LV. Enhanced resin-composite bonding to zirconia framework after pretreatment with selected silane monomers. Dent Mater 2011; 27: 273-280.

10) Matinlinna JP, Lassila LVJ, Vallittu PK. Pilot evaluation of resin composite cement adhesion to zirconia using a novel silane system. Acta Odontol Scand 2007; 65: 44-51.

11) Petty HE, Pickwell R, Osterholtz FD, Huang Su SC. Silicone oligomers and curable compositions containing same. WO 1999054386 A1 1999.

12) Lung CYK, Kukk E, Hägerth T, Matinlinna JP. Surface modification of silica-coated zirconia by chemical treatments. Appl Surf Sci 2010; 257: 1228-1235.

13) Uchida M, Kim HM, Kokubo T, Nawa M, Asano T, Tanaka K, Nakamura T. Apatite-forming ability of a zirconia/alumina nano-composite induced by chemical treatment. J Biomed Mater Res 2002; 60: 277-282.

14) Nishigawa G, Maruo Y, Oka M, Okamoto M, Minagi S, Irie $\mathrm{M}$, Suzuki K. Effect of plasma treatment on adhesion of self- curing repair resin to acrylic denture base. Dent Mater J 2004; 23: 545-549.

15) Nishigawa G, Maruo Y, Irie M, Oka M, Yoshihara K, Minagi S, Nagaoka N, Yoshida Y, Suzuki K. Ultrasonic cleaning of silica-coated zirconia influences bond strength between zirconia and resin luting material. Dent Mater J 2008; 27: 842-848.

16) R Core Team. R: A language and environment for statistical computing. R Foundation for Statistical Computing 2016, Vienna, Austria (URL https://www.R-project.org/)

17) Hooshmand $T$, van Noort $R$, Keshvad A. Storage effect of a pre-activated silane on the resin to ceramic bond. Dent Mater 2004; 20: 635-642.

18) Matinlinna JP, Lassila LVJ, Vallittu PK. The effect of a novel silane blend system on resin bond strength to silica-coated $\mathrm{Ti}$ substrate. J Dent 2006; 34: 436-443.

19) Sarkar D, Mohapatra D, Ray S, Bhattacharyya S, Adak S, Mitra N. Synthesis and characterization of sol-gel derived $\mathrm{ZrO}_{2}$ doped $\mathrm{Al}_{2} \mathrm{O}_{3}$ nanopowder. Cream Int 2007; 33: 12751282.

20) Nishio E, Ikuta N, Okabayashi H. An analytical investigation of silane coupling agents on glass fibres. J Anal Appl Pyrolysis 1991; 18: 261-268.

21) Anagnostopoulos T, Eliades G, Palaghias G. Composition, reactivity and surface interactions of three dental silane primers. Dent Mater 1993; 9: 182-190.

22) Berry T, Barghi N, Chung K. Effect of water storage on the silanization in porcelain repair strength. J Oral Rehabil 1999; 26: 459-463.

23) Aida M, Hayakawa T, Mizukawa K. Adhesion of composite to porcelain with various surface conditions. J Prosthet Dent 1995; 73: 464-470.

24) Kane SR, Ashby PD, Pruitt LA. ATR-FTIR as a thickness measurement technique for hydrated polymer-on-polymer coatings. J Biomed Mater Res B Appl Biomater 2009; 91: 613620.

25) Wu CC, Wei CK, Ho CC, Ding SJ. Enhanced hydrophilicity and biocompatibility of dental zirconia ceramics by oxygen plasma treatment. Materials (Basel) 2015; 8: 684-699.

26) Kern M, Wegner SM. Bonding to zirconia ceramic: adhesion methods and their durability. Dent Mater 1998; 14: 64-71.

27) Atsu SS, Kilicarslan MA, Kucukesmen HC, Aka PS. Effect of zirconium-oxide ceramic surface treatments on the bond strength to adhesive resin. J Prosthet Dent 2006; 95: 430436.

28) Akgungor G, Sen D, Aydin M. Influence of different surface treatments on the short-term bond strength and durability between a zirconia post and a composite resin core material. J Prosthet Dent 2008; 99: 388-399.

29) Tanaka R, Fujishima A, Shibata Y, Manabe A, Miyazaki T. Cooperation of phosphate monomer and silica modification on zirconia. J Dent Res 2008; 87: 666-670.

30) Matsumoto TJ, An SH, Ishimoto T, Nakano T, Matsumoto T, Imazato S. Zirconia-hydroxyapatite composite material with micro porous structure. Dent Mater 2011; 27: e205-212.

31) An SH, Matsumoto T, Miyajima H, Nakahira A, Kim KH, Imazato S. Porous zirconia/hydroxyapatite scaffolds for bone reconstruction. Dent Mater 2012; 28: 1221-1231.

32) Okada M, Matsumoto T. Synthesis and modification of apatite nanoparticles for use in dental and medical applications. Jpn Dent Sci Rev 2015; 51: 85-95.

33) Joulaei M, Bahari M, Ahmadi A, Savadi Oskoee S. Effect of different surface treatments on repair micro-shear bond strength of silica- and zirconia-filled composite resins. J Dent Res Dent Clin Dent Prospects 2012; 6: 131-137.

34) Amirouche-Korichi A, Mouzali M, Watts DC. Effects of monomer ratios and highly radiopaque fillers on degree of conversion and shrinkage-strain of dental resin composites. Dent Mater 2009; 25: 1411-1418. 